Background: Non-Hodgkin B-cell lymphoma (NHL), especially mucosa-associated lymphoid tissue (MALT) lymphoma, is one of the main complications of primary Sjögren's syndrome (pSS). Frequent extranodal lymphoproliferation makes its diagnosis challenging and obtaining a biopsy difficult. Since pSS-associated lymphomas are very frequently MALT lymphomas with salivary gland involvement, we hypothesized that minor salivary gland biopsy (MSGB) could be useful for NHL diagnosis in this context.

Objectives: To evaluate the potential contribution of MSGB for the diagnosis of pSS-associated MALT lymphoma by comparing patients diagnosed with NHL based on MSGB or another tissue.

Methods: All pSS patients (ACR/EULAR 2016 classification criteria), from the Paris National Referral Centers for Rare Systemic Autoimmune Diseases, diagnosed with NHL between January 2010 and October 2019, were included. Each patient's clinical, biological, radiological and therapeutic information was collected retrospectively at NHL diagnosis and 1-year later. Only patients with MSGB available were analyzed; they were divided into 2 groups according to MSGB results for $\mathrm{NHL}^{\mathrm{MSGB}} \mathrm{MS}^{+}$and $\mathrm{MSGB}^{-}$.

Results: Among 36 pSS patients diagnosed with NHL during the study period, 25 had an MSGB available at the time of NHL diagnosis. Among them, 13 MSGBs contained $\mathrm{NHL}\left(\mathrm{MSGB}^{+}\right)$. MSGB was the only site enabling $\mathrm{NHL}$ diagnosis for $10 / 13$ (77\%); pSS and NHL were diagnosed simultaneously in 4/13 (31\%). MSBGs were $\mathrm{NHL}^{-}$for lymphomas diagnosed based on other tissue samples for 12 (48\%) patients (MSGB ${ }^{-}$). The clinical, biological, histological and radiological characteristics of both groups are reported in Table 1. No major differences were found between groups for median ESSDAI at NHL diagnosis and the frequency of salivary gland hypermetabolism on PET-CT. MALT-type NHL was found in 24/25 (96\%) patients including $13 / 13(100 \%)$ of those MSGB $^{+}$and $11 / 12(92 \%)$ of those MSGB ${ }^{-}$. Six of the $13(46 \%) \mathrm{MSGB}^{+}$patients received no treatment, while all $\mathrm{MSGB}^{-}$patients were treated. Between diagnosis and 1 year of follow-up, ESSDAI scores without the NHL item did not differ (6.5 [3.5-9.5]) for the 6 untreated patients, but had significantly decreased for the 19 treated patients $(3.5[2.0-5.8])(p=0.02)$.

Table 1. Comparison of the pSS patients' characteristics according to $\mathrm{MSGB}^{+}$vs. MSGB ${ }^{-}$for NHL

\begin{tabular}{lcc}
\hline Characteristic & MSGB $^{+}, \mathbf{n = 1 3}$ & MSGB $^{-}, \mathbf{n}=\mathbf{1 2}$ \\
\hline Female/male (ratio) & $12 / 1(12)$ & $11 / 1(11)$ \\
Age at NHL diagnosis, yr & $60(52-72)$ & $58(49.5-69.8)$ \\
pSS duration at NHL diagnosis, yr & $2 .(0-9)$ & $3.5(0.8-11)$ \\
ESSDAl score without NHL item & $9(6-16)$ & $10 .(3.5-19.8)$ \\
Cryoglobulinemia ${ }^{+}$ & $9(69)$ & $3 / 9(33)$ \\
Rheumatoid factor & $8 / 8(100)$ \\
Anti-SSA antibody & & $7(58)$ \\
Anemia (Hb<12g/dL) & $10(77)$ & $6 / 11(55)$ \\
Gammaglobulins, g/L & $2(15)$ & $16.1(12.3-20.0)$ \\
Histology & $12.4(10.8-16.9)$ & $11(92)$ \\
$\quad$ MALT-type lymphoma & $13(100)$ & $1(8)$ \\
Diffuse-large B-cell lymphoma & $0(0)$ & $3 / 10(30)$ \\
${ }^{18}$ F]FDG-PET-CT, SUV max $>4.7$ & $6 / 12(50)$ & $3 / 10(30)$ \\
$\quad$ Parotid or submandibular gland & $1 / 12(8)$ & \\
Lymph nodes &
\end{tabular}

Results are presented as number (\%) or median (IQR).

Conclusion: Our results showed that, when MALT lymphoma is suspected, MSGB contributed to diagnosing NHL, either at initial assessment or during pSS evolution, enabling MALT lymphoma diagnosis in at least a third of $\mathrm{NHL}$ patients and $>50 \%$ when MSGB was obtained systematically. Thus, MSGB might avoid the need for a more invasive procedure. Moreover, our findings suggest MSGB should be obtained at pSS diagnosis, and repeatedly during follow-up, when NHL, especially MALT, is suspected.

Disclosure of Interests: Simon Parreau: None declared, Raphaèle Seror Consultant of: BMS UCB Pfizer Roche, Benjamin Terrier: None declared, Barbara Burroni: None declared, Céline Jamart: None declared, Alexis Régent: None declared, Gaetane Nocturne: None declared, Luc Mouthon: None declared, Xavier Mariette Consultant of: BMS, Gilead, Medimmune, Novartis, Pfizer, Servier, UCB, Véronique LE GUERN Grant/research support from: UCB for GR2 study (to our institution)

DOI: 10.1136/annrheumdis-2020-eular.3825

\section{SAT0227 CLINICAL AND IMMUNOLOGICAL PROFILE OF NEUROPSYCHIATRIC LUPUS IN SOUTH INDIA}

B. Pinto ${ }^{1}$, S. $\mathrm{Cs}^{2}$, D. Susanne ${ }^{3}$, R. Aithala ${ }^{4}$, C. Kodishala ${ }^{2}$, B. S. Charles ${ }^{2}$, S. $_{\mathrm{Kn}^{2}}$ R. Nadig ${ }^{5}$, G. R. Sarma ${ }^{5}$, V. Shobha ${ }^{2} .{ }^{1}$ St John's Medical College, Clinical Immunology and Rheumatology, Bangalore, India; ${ }^{2}$ St. John's Medical College, Clinical Immunology and Rheumatology, Bangalore, India; ${ }^{3}$ St. John's Medical College, Radiology, Bangalore, India; ${ }^{4}$ St. John's Medical College, Clinical Immunology and Rheumatology, Bangalore, India; ${ }^{5}$ St. John's Medical College, Neurology, Bangalore, India
Background: Neuropsychiatric manifestations are one of the least understood aspects of SLE.

Objectives: To study the prevalence of NPSLE manifestations in our cohort and compare clinical and immunological features of patients with controls.

Methods: This was a retrospective study in a tertiary care centre. Classification of SLE was as per SLICC criteria. All patients of SLE diagnosed in the last 5 years with NP manifestations as per the ACR definitions were included. Consecutive patients of SLE over last 2 years without NP manifestations were taken as controls.

Results: Six hundred and thirty one patients with SLE were seen in our centre from Jan 2013 to Jun-2019. NPSLE manifestations as per the ACR definitions were seen in 100 patients (95 females) .137 (130 females) consecutive SLE patients without NP manifestations were included as controls. The commonest NPSLE manifestation was seizures followed by cerebrovascular accident (Table 1). NPSLE manifestation occurred at the first presentation in 77 patients. Forty patients had more than one NPSLE manifestation. Antiphosholipid (API) antibodies (lupus anticoagulant and anticardiolipin antibody) were tested in 94 patients and positive in 25 (26.3\%). Only 10 of the 23 patients with CVA were positive for API. NPSLE syndromes were similar in API positive and negative patients. As compared to controls, NPSLE patients had lower prevalence of mucocutaneous and musculoskeletal manifestations. There were no differences in the autoantibody profile in NPSLE patients versus controls. MRI of the brain was available in 65 NP episodes and 51 were abnormal. Ischaemic changes (22) were the commonest followed by T2/FLAIR hyperintensities (16). Cyclophosphamide was used for induction in 46 and Mycophenolate in 19 patients. Follow up of $\geq 6$ months duration was available 93 patients. Over a mean follow up duration of $31.1 \pm 22.7$ months, 6 patiens had a NPSLE relapse and 10 died.

Table 1. Prevalence of NP SLE manifestations $(\mathrm{N}=631)$ and clinical and immunological features

\begin{tabular}{|c|c|c|c|c|c|}
\hline NPSLE manifestations & $\begin{array}{l}\text { Prevalence } \\
(\%)\end{array}$ & Clinical Feature & $\begin{array}{l}\text { NPSLE } \\
(n=100)\end{array}$ & $\begin{array}{l}\text { Controls } \\
(n=137)\end{array}$ & $\begin{array}{c}P \\
\text { value }\end{array}$ \\
\hline All & $100(15.85 \%)$ & Age & $29.1 \pm 11.6$ & $28.18 \pm 9.79$ & NS \\
\hline Central Nervous System & $65(10.3 \%)$ & $\mathrm{M}: \mathrm{F}$ & $5: 95$ & $7: 130$ & NS \\
\hline Aseptic meningitis & $2(0.3 \%)$ & Mucocutaneous & $55(55 \%)$ & $105(76.6 \%)$ & $<0.001$ \\
\hline Acute Confusional State & $12(1.9 \%)$ & Musculoskeletal & $57(57 \%)$ & $96(70 \%)$ & 0.03 \\
\hline Cerebrovascular Disease & $23(3.6 \%)$ & Hemolytic Anaemia & $19(19 \%)$ & $40(29.2 \%)$ & NS \\
\hline Cognitive Dysfunction & $2(0.3 \%)$ & Leucopenia & $25(25 \%)$ & $40(29.2 \%)$ & NS \\
\hline Demyelinating Syndrome & $3(0.5 \%)$ & Thrombocytopenia & $22(22 \%)$ & $36(26.3 \%)$ & NS \\
\hline Headache & $12(1.9 \%)$ & Serositis & $10(10 \%)$ & $16(11.7 \%)$ & NS \\
\hline Movement Disorder & $7(1.1 \%)$ & Lupus Nephritis & $41(41 \%)$ & $38(27.7 \%)$ & NS \\
\hline Myelopathy & $5(0.8 \%)$ & Direct Coomb's Test & $44(44 \%)$ & $55(45.5 \%)$ & NS \\
\hline Seizures & $35(5.5 \%)$ & $\begin{array}{l}\text { Lupus anticoagulant } \\
\text { Anticardiolipin }\end{array}$ & $\begin{array}{l}15(15 \%) \\
12(12 \%)\end{array}$ & $\begin{array}{l}21(18.6 \%) \\
14(12.5 \%)\end{array}$ & $\begin{array}{l}\text { NS } \\
\text { NS }\end{array}$ \\
\hline $\begin{array}{l}\text { Peripheral Nervous } \\
\text { system }\end{array}$ & $28(4.4 \%)$ & Low complements & $78(78 \%)$ & $98(78.4 \%)$ & NS \\
\hline AIDP & $3(0.5 \%)$ & dsDNA & $44(44 \%)$ & $67(52.8 \%)$ & NS \\
\hline Autonomic Disorders & $1(0.1 \%)$ & Smith & $38(38 \%)$ & $51(40.8 \%)$ & NS \\
\hline Cranial Neuropathy & $5(0.8 \%)$ & Nucleosome & $43(43 \%)$ & $52(41.9 \%)$ & NS \\
\hline Mono neuropathy & $9(1.4 \%)$ & Histone & $27(27 \%)$ & $40(32.3 \%)$ & NS \\
\hline Myasthenia Gravis & 0 & Ribo P & $25(25 \%)$ & $37(29.6 \%)$ & NS \\
\hline Plexopathy & 0 & $\mathrm{RNP} / \mathrm{Sm}$ & $43(43 \%)$ & $52(41.6 \%)$ & NS \\
\hline \multirow[t]{2}{*}{ Polyneuropathy } & $12(1.9 \%)$ & scl70 & $4(4 \%)$ & $5(4 \%)$ & NS \\
\hline & & SSA & $34(34 \%)$ & $42(33.6 \%)$ & NS \\
\hline Psychiatric & $17(2.7 \%)$ & SSB & $10(10 \%)$ & $18(14.5 \%)$ & NS \\
\hline Mood Disorders & $8(1.3 \%)$ & Ro52 & $32(32 \%)$ & $39(31.2 \%)$ & NS \\
\hline Psychosis & $13(2.1 \%)$ & $\mathrm{PmScl}$ & 0 & $5(4 \%)$ & NS \\
\hline Anxiety Disorder & $4(0.6 \%)$ & PCNA & $2(2 \%)$ & $6(4.8 \%)$ & NS \\
\hline
\end{tabular}

Conclusion: Seizures and CVA are the commonest NPSLE syndromes. API positivity was not associated with NPSLE in our cohort.

Disclosure of Interests: None declared

DOI: 10.1136/annrheumdis-2020-eular.4010

\section{\begin{tabular}{|l|l}
\hline SAT0228 & PREGNANCY OUTCOME IN SYSTEMIC LUPUS \\
\hline
\end{tabular} ERYTHEMATOSUS: A MONOCENTRIC COHORT ANALYSIS}

C. Pirone ${ }^{1}$, F. Ceccarelli ${ }^{1}$, A. Selntigia ${ }^{2}$, C. Perricone ${ }^{3}$, S. Truglia ${ }^{1}$, V. A. Pacucci ${ }^{1}$, F. R. Spinelli ${ }^{1}$, C. Alessandri ${ }^{1}$, G. Valesini ${ }^{1}$, G. Perrone ${ }^{2}$, F. Conti ${ }^{1} .{ }^{1}$ Lupus Clinic, Reumatologia, Sapienza Università di Roma, Roma, Italy; ${ }^{2}$ Dipartimento di Scienze Ginecologiche-Ostetriche e Urologiche, Roma, Italy; ${ }^{3}$ Reumatologia, Università di Perugia, Perugia, Italy

Background: Systemic Lupus Erythematosus (SLE) is a chronic autoimmune disease, affecting prevalently women in childbearing age. Thanks to pre-gestational counseling and multi-disciplinary approach, adopted in daily clinical practice, SLE patients are experiencing even more uncomplicated pregnancies.

Objectives: Here, we evaluated pregnancy outcome in a large SLE cohort, compared to a control group including pregnant women without autoimmune diseases. 